\title{
Analysis and Design of Output Feedback PD Controllers for Time-Delay Systems
}

\author{
Pieter Appeltans ${ }^{1}$, Silviu-Iulian Niculescu ${ }^{2}$ and Wim Michiels ${ }^{1}$
}

\begin{abstract}
In this paper we analyze proportional-derivative output feedback control for single-input single-output linear time-invariant dynamical systems with discrete state delays. The main focus of the presented work is the analysis of the effect of arbitrarily small implementation errors, such as a feedback delay or a finite-difference approximation of the derivative, on the stability of the closed loop. More specifically, it will be shown that the classical notion of stability does not suffice to adequately describe the asymptotic behavior of the closed loop system in the presence of these implementation errors, as stability might be lost even when these errors are made arbitrarily small. This paper therefore considers strong stability, which eliminates this well-posedness problem by not only requiring stability but by also enforcing that stability is preserved for sufficiently small perturbations. To conclude this paper, a controller design methodology for proportionalderivative output feedback controllers that strongly stabilize the system, is proposed.
\end{abstract}

\section{INTRODUCTION}

Even for systems without state delays, it is well-known that the stability properties of derivative feedback controllers might be sensitive to infinitesimal perturbations [1]. In such a context it thus not suffices to only guarantee stability, as arbitrarily small implementation errors might render the real-life realization of the system unstable. Both [2] and [1] therefore introduced a robustified notion of stability, $w$ stability in [2] and p-stability in [1], which not only requires the stability of the closed-loop system, but also that stability is preserved under sufficiently small perturbations. Another common feature of both works, is that they both suggest that the stability of the closed loop system can be preserved under sufficiently small implementation errors by adding a high frequency roll-off to the control loop, given that certain conditions on the nominal closed loop system are fulfilled. In this work, we will examine similar sensitivity of stability effects for proportional-derivative (PD) output feedback control of linear time-invariant (LTI), single-input single-output (SISO) systems with state delays. An extended version of

\footnotetext{
*This work was supported by the project C14/17/072 of the KU Leuven Research Council and by the project G0A5317N of the Research Foundation-Flanders (FWO - Vlaanderen). The authors are also members of the International Research Network (IRN) Distributed Parameter Systems with Constraints ("Spa-DisCo") financially supported by the French CNRS and various European institutions and universities (including KU Leuven, Belgium) for the period 2018-2021.

1 Department of Computer Science, KU Leuven, Leuven, Belgium (Pieter.Appeltans@kuleuven.be, Wim.Michielsakuleuven.be)

${ }^{2}$ Laboratory of Signals and Systems (L2S), Inria Team "DISCO", CNRS, CentraleSupélec, Université Paris-Saclay, Gif-sur-Yvette, France (Silviu.Niculescull2s.centralesupelec.fr)
}

the theoretical part of this paper, discussing proportionalintegral-derivative (PID) output feedback control of multipleinput multiple-output (MIMO) time-delay systems, can be found in [3].

The effect of arbitrarily small implementation errors on the stability of dynamical systems is widely studied in the field of systems and control theory. For example, [4, p. 87101] examines the effect of introducing small time delays in the control loop on the stability of the closed loop system. Inspired by that work, this effect is further examined in [5], [6] and [7]. Another example are delay differential equations of neutral type for which it is well-known that the stability of the null solution might be sensitive to arbitrarily small perturbations on the delays. The authors of [8] therefore introduced the notion of strong stability, which requires that stability is preserved under sufficiently small delay changes. Next, [9] and [10] examine the effect of sufficiently small perturbations on the closed loop stability of singularly perturbed systems. Finally, [11] discusses the effect of an arbitrarily close derivative approximation on gradient play dynamics.

Our choice for PD controllers is motivated by their improved transient performance compared to proportional controllers. Such PD controllers are therefore frequently used in teleoperation [12] and haptics [13].

As mentioned above, in this work we restrict our attention to LTI SISO systems of the following form

$$
\left\{\begin{array}{l}
\dot{x}(t)=A_{0} x(t)+\sum_{k=1}^{K} A_{k} x\left(t-\tau_{k}\right)+b u(t) \\
y(t)=c^{T} x(t)
\end{array}\right.
$$

with $x \in \mathbb{R}^{n}$ the internal state, $u \in \mathbb{R}$ the input, $y \in \mathbb{R}$ the output, $0<\tau_{1}<\cdots<\tau_{K}<+\infty$ discrete delays, $b \in \mathbb{R}^{n}$ the input vector, $c \in \mathbb{R}^{n}$ the output vector, and $A_{0}, A_{1}, \ldots$, $A_{K-1}$ and $A_{K} \in \mathbb{R}^{n \times n}$ the state matrices. The goal is to design a PD output feedback controller,

$$
u(t)=k_{p} y(t)+k_{d} \dot{y}(t)
$$

with $k_{p}, k_{d} \in \mathbb{R}$, that (asymptotically) stabilizes the system. By transforming (1) and (2) to the Laplace domain, we can examine the asymptotic behavior of the closed loop system using the following (non-linear) characteristic function

$$
\begin{aligned}
\Delta(\lambda):=\operatorname{det}\left(\left(I_{n}-k_{d} b c^{T}\right) \lambda-A_{0}\right. \\
\left.-\sum_{k=1}^{K} A_{k} e^{-\lambda \tau_{k}}-k_{p} b c^{T}\right) .
\end{aligned}
$$


More specifically, the closed loop system of (1) and (2) is asymptotically stable if (and only if) all roots of (3) lie in the open left half-plane.

However, as shown in the next section, the stability of this closed loop system might be sensitive to arbitrarily small implementation errors such as feedback delay, a finite difference approximation of the derivative or low-pass filtering of the feedback signal. To address this sensitivity of stability problem, [3] introduced the notion of strong stability, inspired by the notion of strong stability for delay differential equations of neutral type, which requires that stability is preserved for sufficiently small implementation errors. Note that this definition for strong stability differs from the one introduced in [14, Section 5.3], which refers to stabilization using a controller that is itself stabilizing.

The remainder of this work is structured as follows. Section II examines several implementation errors that render a stable closed loop system unstable even when made arbitrarily small. In Section III, we recall the definition of strong stability from [3] and give conditions on the feedback gains for which this can be achieved. In Section IV the presented results are illustrated using two small dimensional timedelay systems. Section V discusses a design methodology for PD output feedback controllers that are strongly stabilizing. Finally, Section VI draws some concluding remarks.

\section{LOSING STABILITY DUE TO ARBITRARILY SMALL PERTURBATIONS}

In this section we show that under certain conditions on the derivative feedback gain $k_{d}$, a stable closed loop of (1) and (2) loses stability under an arbitrarily small feedback delay (Subsection II-A), an arbitrarily close finite difference approximation of the derivative (Subsection II-B) and low-pass filtering of the derivative feedback signal with an arbitrarily large cut-off frequency (Subsection II-C). The presented results are a simplification of the results obtained for the PID control of MIMO systems examined in [3]. We refer to that paper for more details and more detailed versions of the presented proofs.

\section{A. Feedback delay}

As first implementation error, we consider a delay in the feedback loop. Shifting this delay to the input, (1) becomes

$$
\left\{\begin{array}{l}
\dot{x}(t)=A_{0} x(t)+\sum_{k=1}^{K} A_{k} x\left(t-\tau_{k}\right)+b u(t-r), \\
y(t)=c^{T} x(t)
\end{array}\right.
$$

with $r>0$ an arbitrarily small positive delay. The following proposition shows that under a certain condition on $k_{d}$, a controller of form (2) that is stabilizing for system (1), may no longer be stabilizing for system (4) even if the feedback delay $r$ is arbitrarily small.

Proposition 1. Assume that the feedback gains $k_{p}$ and $k_{d}$ asymptotically stabilize (1)-(2). If $\left|k_{d} c^{T} b\right|>1$, then the closed loop of (4) and (2) is unstable for all $r>0$.
Proof. The behavior of the closed loop of (4) and (2) is described by the following delay differential equation

$$
\begin{aligned}
& \dot{x}(t)-k_{d} b c^{T} \dot{x}(t-r)= \\
& A_{0} x(t)+\sum_{k=1}^{K} A_{k} x\left(t-\tau_{k}\right)+k_{p} b c^{T} x(t-r),
\end{aligned}
$$

which is of neutral type. It follows from [15, Proposition 1.25] that the closed loop system is unstable independent of $r>0$ if $\rho\left(k_{d} b c^{T}\right)=\left|k_{d} c^{T} b\right|>1$.

\section{B. Finite difference approximation of the derivative}

In some applications, not all state derivatives can be measured. Under those circumstances, one has to resort to a finite difference approximation:

$$
\dot{y}(t) \approx \frac{y(t)-y(t-r)}{r} .
$$

The PD output feedback signal now becomes

$$
u(t)=k_{p} y(t)+k_{d} \frac{y(t)-y(t-r)}{r},
$$

with $r>0$ a sufficiently small positive delay. The following proposition show that also an arbitrarily close finite difference approximation can destroy the stability of the closed loop for certain values of $k_{d}$.

Proposition 2. Assume that the feedback gains $k_{p}$ and $k_{d}$ asymptotically stabilize (1)-(2). If $k_{d} c^{T} b>1$, then there exists a constant $\hat{r}>0$ such that the closed loop of (1) and (5) is unstable for all $r \in(0, \hat{r})$.

Proof. The stability of the closed loop of (1) and (5) is characterized by the solutions of

$$
\begin{aligned}
\Delta_{f d}(\lambda ; r):=\operatorname{det} & \left(\lambda I_{n}-k_{d} b c^{T} \frac{1-e^{-\lambda r}}{r}\right. \\
& \left.-A_{0}-\sum_{k=1}^{K} A_{k} e^{-\lambda \tau_{k}}-k_{p} b c^{T}\right)=0 .
\end{aligned}
$$

Multiplying this equation by $r^{n}$ and introducing the change of variables $\mu=\lambda r$ gives

$$
\begin{aligned}
\operatorname{det}\left(\mu I_{n}-\right. & k_{d} b c^{T}\left(1-e^{-\mu}\right) \\
& \left.-r A_{0}-\sum_{k=1}^{K} r A_{k} e^{-\mu \tau_{k} / r}-r k_{p} b c^{T}\right)=0 .
\end{aligned}
$$

As $r \searrow 0$, the left-hand side of this equation uniformly converges to the function

$$
\tilde{\Delta}_{f d}(\mu)=\operatorname{det}\left(\mu I_{n}-k_{d} b c^{T}\left(1-e^{-\mu}\right)\right)
$$

on compact regions in the open right half-plane. It is easy to show that $\tilde{\Delta}_{f d}(\mu)$ has at least one root in the open right half-plane if $k_{d} c^{T} b>1$, see for example [16]. Next, as in the proofs of [3, Proposition 3.2] and [1, Proposition 3.1], one can use the uniform convergence of the left-hand side of (6) to $\tilde{\Delta}_{f d}(\mu)$ on compact regions in the right half-plane and Rouché's theorem [17] to show that there exist $\epsilon, \hat{r}>0$ such that $\Delta_{f d}(\lambda ; r)$ has at least one root in the right half-plane $\{\lambda \in \mathbb{C}: \Re(\lambda)>\epsilon / r\}$ for all $r \in(0, \hat{r})$. 


\section{Low pass filtering}

As a final example of a perturbation that can cause a loss of stability even when made infinitesimal, we examine low pass filtering of the derivative feedback signal. The feedback signal now becomes

$$
u(t)=k_{p} y(t)+k_{d} \zeta(t)
$$

with

$$
T \dot{\zeta}(t)+\zeta(t)=\dot{y}(t)
$$

and $1 / T$ the cut-off frequency of the filter.

Proposition 3. Assume that the feedback gains $k_{p}$ and $k_{d}$ asymptotically stabilize (1)-(2). If $k_{d} c^{T} b>1$, then there exists a constant $\hat{T}>0$ such that the closed-loop of (1) and (7) is unstable for all $T \in(0, \hat{T})$. On the other hand, if $k_{d} c^{T} b<1$, then there exists a constant $\tilde{T}>0$ such that the closed loop of (1) and (7) is asymptotically stable for all $T \in(0, \tilde{T})$.

Proof. The asymptotic stability of the closed loop of (1) and (7) is characterized by the roots of

$$
\begin{aligned}
\Delta_{l p}(\lambda ; T):=\operatorname{det}( & \left(I_{n}-k_{d} b c^{T} \frac{1}{1+\lambda T}\right) \lambda \\
& \left.\quad-A_{0}-\sum_{k=1}^{K} A_{k} e^{-\lambda \tau_{k}}-k_{p} b c^{T}\right) .
\end{aligned}
$$

Multiplying this function with $T^{n}$ and introducing the change of variable $\mu=\lambda T$ results in

$$
\begin{aligned}
\bar{\Delta}_{l p}(\mu ; T):= & \operatorname{det}\left(\left(I_{n}-k_{d} b c^{T} \frac{1}{1+\mu}\right) \mu\right. \\
& \left.-T A_{0}-\sum_{k=1}^{K} T A_{k} e^{-\mu \tau_{k} / T}-T k_{p} b c^{T}\right) .
\end{aligned}
$$

As $T \searrow 0$, this function uniformly converges to

$$
\tilde{\Delta}_{l p}(\mu):=\operatorname{det}\left(\mu I_{n}-k_{d} b c^{T} \frac{\mu}{1+\mu}\right)
$$

on compact regions in the open right-half plane. This last function has a root in the open right-half plane if (and only if) $k_{d} c^{T} b>1$. Also in this case, one can use the uniform convergence of $\bar{\Delta}_{l p}(\mu ; T)$ to $\tilde{\Delta}_{l p}(\mu)$ on compact regions in the open right half-plane for $T \searrow 0$ and Rouché's theorem to show that this implies that there exist $\epsilon, \widehat{T}>0$ such that $\Delta_{l p}(\lambda ; T)$ has a root with real part larger than $\epsilon / T$ for all $T \in(0, \hat{T})$.

On the other hand, if $k_{d} c^{T} b<1$, then there exist $\epsilon, \bar{T}>0$ such that $I-k_{d} b c^{T} \frac{1}{\lambda T+1}$ is invertible for all $\lambda$ in the half plane $V:=\{\lambda \in \mathbb{C}: \Re(\lambda)>-\epsilon\}$ and all $T \in(0, \bar{T})$. This means that $\Delta_{l p}(\lambda ; T)=0$ on $V$ can be rewritten as

$$
\begin{aligned}
& \operatorname{det}\left(I_{n} \lambda-\left(I_{n}-k_{d} b c^{T} \frac{1}{1+\lambda T}\right)^{-1} \times\right. \\
&\left.\left(A_{0}+\sum_{k=1}^{K} A_{k} e^{-\lambda \tau_{k}}+k_{p} b c^{T}\right)\right)=0 .
\end{aligned}
$$

Thus, for $T \in(0, \bar{T})$, if $\Delta_{l p}(\lambda ; T)$ has a characteristic root, $\lambda_{0}$, inside $V$, it must be bounded in modulus by

$$
\begin{aligned}
\left|\lambda_{0}\right| \leq \sup _{\lambda \in V}\left\|\left(I_{n}-k_{d} b c^{T} \frac{1}{1+\lambda T}\right)^{-1}\right\| \times \\
\left(\left\|A_{0}+k_{p} b c^{T}\right\|+\sum_{k=1}^{K}\left\|A_{k}\right\| e^{-\Re(\lambda) \tau_{k}}\right)<\infty
\end{aligned}
$$

The eigenvalues of $\Delta_{l p}(\lambda ; T)$ in $V$ lie thus in a compact region and $\Delta_{l p}(\lambda ; T)$ uniformly converges to $\Delta(\lambda)$ on such compact regions as $T \searrow 0$. Because $\Delta(\lambda)$ is stable, we can choose $\epsilon$ such that $\Delta(\lambda)$ has no roots in $V$. Combining this information with Rouché's theorem as in the proof of [3, Proposition 4.1], one can show that there exists a constant $\tilde{T}>0$ such that $\Delta_{l p}(\lambda ; T)$ has no roots in the aforementioned compact region and hence in $V$. Stability is thus preserved, for all $T \in(0, \tilde{T})$.

\section{STRONG STABILITY}

\section{A. Definition}

As seen in the previous section, the notion of (asymptotic) stability does not suffice to adequately describe the behavior of the closed loop system with PD output feedback control as stability might be lost under arbitrarily small implementation errors. Therefore, [3, Section 4.2] introduced the concept of strong stability, which requires the closed loop system not only to be stable but also to preserve this stability under a certain class of sufficiently small perturbations. More specifically, it considers the perturbation framework depicted in Figure 1, in which the functions $R_{1}\left(\lambda ; r_{1}\right), R_{2}\left(\lambda ; r_{2}\right)$ and $R\left(\lambda ; r_{3}\right)$ represent the perturbations. These perturbation functions are required to fulfill the conditions given in Assumption 1. The additional low pass filter (dashed box) will come into play in the next subsection.

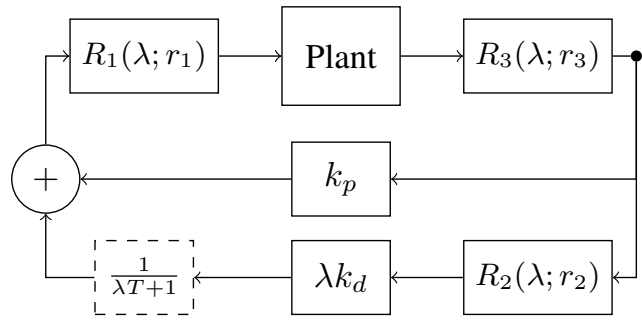

Fig. 1: Closed loop description of the considered perturbation framework.

Assumption 1. The perturbations $R_{1}\left(\lambda ; r_{1}\right), R_{2}\left(\lambda ; r_{2}\right)$ and $R\left(\lambda ; r_{3}\right)$ fulfill the following conditions

1) for every $r \geq 0$, the functions $\left\{\lambda \mapsto R_{i}(\lambda ; r)\right\}_{i=1}^{3}$ are meromorphic and for every $\lambda \in \mathbb{C}$, the functions $\left\{r \mapsto R_{i}(\lambda ; r)\right\}_{i=1}^{3}$ are continuous;

2) $\lambda \mapsto R_{i}(\lambda ; 0) \equiv 1$ for $i=1,2,3$;

3) for every compact set $\Omega \subset \mathbb{C}$, we have

$$
\lim _{r \rightarrow 0+} \max _{\lambda \in \Omega}\left|R_{i}(\lambda ; r)-1\right|=0 \text { for } i=1,2,3
$$

i.e. the functions $\left\{\lambda \mapsto R_{i}(\lambda ; r)\right\}_{i=1}^{3}$ uniformly converge to the identity function on compact regions in the complex plane as $r$ goes to zero;

4) there exist constants $M, N, \hat{r}>0$ such that for all $\lambda \in \mathbb{C}$ with $\Re(\lambda) \geq-N$ and for all $r \in(0, \hat{r})$

$$
\left|R_{i}(\lambda ; r)\right| \leq M \text { for } i=1,2,3 .
$$

The implementation errors studied in Section II fit this framework: $R_{1}(\lambda ; r)=e^{-\lambda r}$ and $R_{2}(\lambda ; r)=R_{3}(\lambda ; r)=$ 
1 for (4); $R_{1}(\lambda ; r)=R_{3}(\lambda, r)=1$ and $R_{2}(\lambda, r)=$ $\left\{\begin{array}{ll}\frac{1-e^{-\lambda r}}{\lambda r} & \lambda r \neq 0 \\ 1 & \lambda r=0\end{array}\right.$ for (5); and $R_{1}(\lambda ; r)=R_{3}(\lambda ; r)=1$ and $R_{2}(\lambda ; T)=1 /(\lambda T+1)$ for (7).

Based on these perturbation functions, [3] introduced the following definition for strong stability.

Definition 1. [3, Definition 4.3] A closed loop system as depicted in Figure 1, is strongly stable if it is asymptotically stable and if for every trio of functions $\left\{R_{i}\left(\lambda ; r_{i}\right)\right\}_{i=1}^{3}$ that satisfies the assumptions above, there exists a constant $\hat{r}>0$ such that the closed loop system remains asymptotically stable under these perturbations for all $r_{1}, r_{2}$ and $r_{3}$ in the open interval $(0, \hat{r})$.

\section{B. Guaranteeing strong stability using low pass filtering}

In Section II it was shown that arbitrarily small implementation errors can cause a loss of stability of the closed loop system. In the Laplace domain, this corresponded with characteristic roots with a large real part appearing in the right half-plane via infinity. A natural way to cancel these roots is to add a low pass filter to the control loop, as suggested in [1]. However, from Section II-C it follows that this low pass filter itself might be destabilizing if $k_{d} c^{T} b>1$. Luckily, the following proposition shows that if $k_{d} c^{T} b<1$ then the closed loop system can be made strongly stable by adding a low pass filter with a sufficiently large cut-off frequency (depicted with a dashed box in Figure 1).

Proposition 4. Assume that the feedback gains $k_{p}$ and $k_{d}$ asymptotically stabilize (1)-(2). If $k_{d} c^{T} b<1$, then the closed-loop of (1) and (7) is strongly stable for sufficiently small $T$.

Proof. By considering the perturbations and the low pass filtering of the derivative signal, the characteristic function becomes

$$
\begin{aligned}
\Delta_{R, l p}\left(\lambda ; r_{1}, r_{2}, r_{3}, T\right) & :=\operatorname{det}\left(\left(I_{n}-k_{d} b c^{T} \times\right.\right. \\
& \left.\frac{1}{\lambda T+1} R_{1}\left(\lambda ; r_{1}\right) R_{2}\left(\lambda ; r_{2}\right) R_{3}\left(\lambda ; r_{3}\right)\right) \lambda-A_{0}- \\
& \left.\sum_{k=1}^{K} A_{k} e^{-\lambda \tau_{k}}-k_{p} b c^{T} R_{1}\left(\lambda ; r_{1}\right) R_{3}\left(\lambda ; r_{3}\right)\right) .
\end{aligned}
$$

We choose $M, N$ and $\hat{r}$ according to Assumption 1.4. The characteristic roots of $\Delta_{R, l p}\left(\lambda ; r_{1}, r_{2}, r_{3}, T\right)$ in the half-plane $V=\{\lambda \in \mathbb{C}: \Re(\lambda)>-N\}$ for $r_{1}, r_{2}$ and $r_{3}$ in the interval $(0, \hat{r})$ are then bounded in modulus by

$$
\begin{array}{r}
\left|\lambda_{0}\right| \leq \sup _{\lambda \in V}\left|\frac{\lambda}{\lambda T+1}\right| \cdot\left|k_{d}\right| \cdot\|b\| \cdot\|c\| \cdot M^{3}+\left\|A_{0}\right\|+ \\
\sum_{k=1}^{K}\left\|A_{k}\right\| e^{-\Re(\lambda) \tau_{k}}+\left|k_{p}\right| \cdot\|b\| \cdot\|c\| \cdot M^{2}<\infty .
\end{array}
$$

Furthermore, $\Delta_{R, l p}\left(\lambda ; r_{1}, r_{2}, r_{3}, T\right)$ converges uniformly to $\Delta_{l p}(\lambda ; T)$ for $r_{1}, r_{2}, r_{3} \searrow 0$. Moreover, if $k_{d} c^{T} b<1$, it follows from Proposition 3 that for sufficiently small $T$ there exists an $\epsilon>0$ such that $\Delta_{l p}(\lambda ; T)$ has no root with $\Re(\lambda)>-\epsilon$. Using once again Rouché's theorem, it follows that for sufficiently small $T$ there exists a $0<$ $\tilde{r} \leq \hat{r}$ such that $\Delta_{R, l p}\left(\lambda ; r_{1}, r_{2}, r_{3}, T\right)$ has no root with
$\Re(\lambda)>-\min (\epsilon, N)$ for $r_{1}, r_{2}$ and $r_{3}$ in the open interval $(0, \tilde{r})$.

\section{Necessary condition for strong stability}

The following proposition induces a necessary condition for the existence of feedback gains $k_{p}$ and $k_{d}$ such that the closed loop of (1) and (7) can be made strongly stable for sufficiently large cut-off frequencies.

Proposition 5. If $\Delta(\lambda)$, as defined in (3), has an odd number of roots in the closed right half-plane for $k_{d}=0$ independent of $k_{p}$, then there do not exist feedback gains $k_{p}$ and $k_{d}$ such that the closed loop of (1) and (7) is strongly stable.

Proof. Assume that $k_{p}$ and $k_{d}$ are such that the closed loop of (1) and (2) is asymptotically stable, i.e. (3) has no roots in the closed right half-plane, and such that for $k_{d}=0$ the closed loop is unstable and (3) has an odd number of roots in the closed right half-plane. Now consider the feedback law $u(t)=k_{p} y(t)+\alpha k_{d} \dot{y}(t)$. From our choices for $k_{p}$ and $k_{d}$, it follows that for $\alpha=0$ the corresponding characteristic function has an odd number of roots in the closed right halfplane while for $\alpha=1$ there are none (as the system is stable). Because the roots of the characteristic function move in complex conjugate pairs as $\alpha$ varies from 0 to 1 , this implies that at least one root has to pass through either the origin or appear via infinity when decreasing $\alpha$ to zero. A root passing through the origin is however not possible as a root in the origin would be invariant with respect to $\alpha$. The root must thus appear from infinity. This implies that there exists an $\bar{\alpha} \in(0,1)$ such that $I-\bar{\alpha} k_{d} b c^{T}$ is not invertible, which means that $k_{d} c^{T} b>1$. For each stabilizing controller pair $\left(k_{p}, k_{d}\right)$ it holds that $k_{d} c^{T} b>1$ and thus is the low pass filter itself destabilizing for small $T$.

\section{ILlustration}

In this section we illustrate the results of the two previous sections using two low order examples. Firstly, consider the following third order system:

$$
\left\{\begin{aligned}
\dot{x}(t)= & {\left[\begin{array}{ccc}
0 & 1 & 0 \\
0 & 0 & 1 \\
-1 & 0 & 0
\end{array}\right] x(t)+\left[\begin{array}{ccc}
0 & 0 & 0 \\
0 & 0 & 0 \\
2.15 & 0 & 0
\end{array}\right] x(t-6) } \\
& +\left[\begin{array}{c}
0 \\
0 \\
-1
\end{array}\right] u(t), \\
y(t)= & {\left[\begin{array}{lll}
8.7 & 0 & 1
\end{array}\right] x(t) . }
\end{aligned}\right.
$$

The open loop system is unstable and the associated characteristic function has five roots in the closed right half-plane. The stability of the closed loop with (2) is characterized by the zeros of

$$
\left(1+k_{d}\right) \lambda^{3}+k_{p} \lambda^{2}+8.7 k_{d} \lambda+1+8.7 k_{p}-2.15 e^{-6 \lambda}
$$

Figure 2 shows the number of right half-plane roots of this characteristic quasi-polynomial in function of $k_{p}$ and $k_{d}$ (encircled). This figure can be understood as follows. As $k_{d}$ is decreased from $k_{d}>-1$ to $k_{d}<-1$ a right half-plane root appears $\left(k_{p}>0\right)$ or disappears $\left(k_{p}<0\right)$ via infinity. 
Other changes to the number of right half-plane roots are caused by characteristic roots crossing the imaginary axis. These crossings correspond to characteristic roots $\lambda=\jmath \omega$ for which

$$
\begin{aligned}
& -\jmath\left(1+k_{d}\right) \omega^{3}-k_{p} \omega^{2}+\jmath 8.7 k_{d} \omega+1 \\
& +8.7 k_{p}-2.15 \cos (6 \omega)+\jmath 2.15 \sin (6 \omega)=0 .
\end{aligned}
$$

By splitting the real and imaginary part of this equation,

$$
\begin{array}{r}
-k_{p} \omega^{2}+1+8.7 k_{p}-2.15 \cos (6 \omega)=0 \\
-\left(1+k_{d}\right) \omega^{3}+8.7 k_{d} \omega+2.15 \sin (6 \omega)=0
\end{array}
$$

it is clear that we have a crossing at the origin for $k_{p}=$ $1.15 / 8.7$ and a crossing complex conjugate pair $\pm \jmath \omega$ for

$$
\begin{aligned}
& k_{p}=(2.15 \cos (6 \omega)-1) /\left(8.7-\omega^{2}\right), \\
& k_{d}=\left(\omega^{3}-2.15 \sin (6 \omega)\right) /\left(8.7 \omega-\omega^{3}\right) .
\end{aligned}
$$

We observe that there are two parameter regions for which the closed loop system is asymptotically stable. A large one in the left bottom corner of Figure 2a with $k_{d}<-1$ (dark gray) and a smaller one in the parameter region on which Figure $2 \mathrm{~b}$ focuses (light gray). Parameter pairs $\left(k_{p}, k_{d}\right)$ in the stabilizing region with $k_{d}<-1$, result in controllers that stabilize the nominal system, but for which stability is lost under arbitrarily small perturbations such as those encountered in Section II. Furthermore, the closed loop system can not be made strongly stable by including low pass filtering of the derivative signal as the filter is itself destabilizing. On the other hand, feedback parameters in the light gray region, result in stabilizing controllers that can be made strongly stable by adding a low pass filter of form (8) with a sufficiently large cut-off frequency to the control loop.

Finally, we illustrate the applicability of Proposition 5. Consider the following system of order two

$$
\left\{\begin{array}{l}
\dot{x}(t)=\left[\begin{array}{ll}
0 & 1 \\
0 & 0
\end{array}\right] x(t)+\left[\begin{array}{ll}
0 & 0 \\
2 & 0
\end{array}\right] x(t-6)+\left[\begin{array}{l}
0 \\
1
\end{array}\right] u(t), \\
y(t)=\left[\begin{array}{ll}
0 & 1
\end{array}\right] x(t) .
\end{array}\right.
$$

It can be verified that the open loop system is unstable and that the associated characteristic function $\left(\lambda^{2}-2 e^{-6 \lambda}\right)$ has three roots in the closed right half plane. The characteristic function corresponding to the closed loop with (2) is

$$
\left(1-k_{d}\right) \lambda^{2}-2 e^{-6 \lambda}-k_{p} \lambda,
$$

which for $k_{d}=0$ reduces to $\lambda^{2}-2 e^{-6 \lambda}-k_{p} \lambda$. By varying $k_{p}$ the number of right half-plane roots of this last function can only change by roots crossing the imaginary axis in complex conjugate pair as a root passing through either the origin or via infinity is impossible. As for $k_{p}=0$ there are three roots in the closed right half plane, the number of right half-plane roots of $\lambda^{2}-2 e^{-6 \lambda}-k_{p} \lambda$ must thus remain odd, independent of $k_{p}$. It now follows from Proposition 5 that the system can not be strongly stabilized with PD output feedback control and low pass filtering of the derivative signal as there do not exist controller parameters $k_{p}$ and $k_{d}$ such that (12) has no right half plane roots and $k_{d} c^{T} b<1$.

This can be verified using Figure 3, which shows the number of right half-plane roots of (12) in function of $k_{p}$

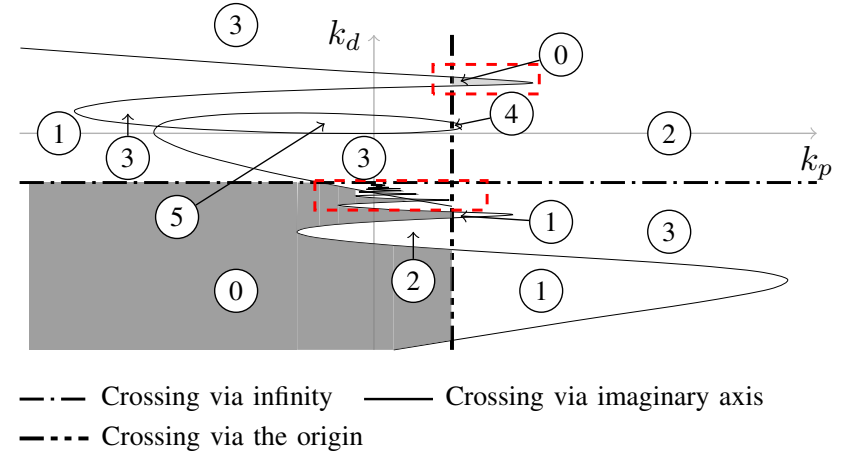

(a) $\left(k_{p}, k_{d}\right) \in[-0.6,0.75] \times[-4.4,2]$
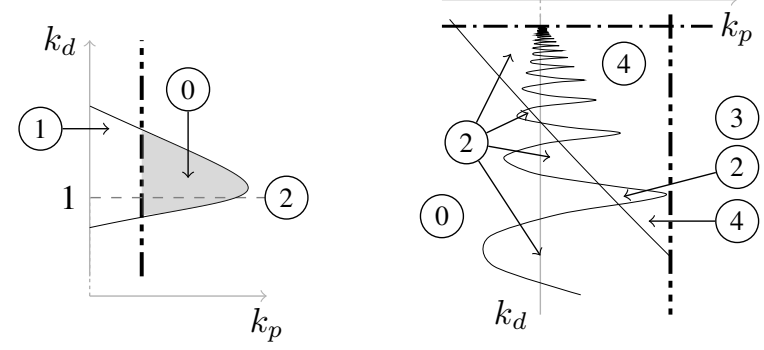

(b) $\left(k_{p}, k_{d}\right) \in[0,0.3] \times[0.8,1.3] \stackrel{(\mathrm{c})}{\left(k_{p},\right.}$

$\left(k_{p}, k_{d}\right) \in[-0.1,0.2] \times[0.96,-1.6]$

Fig. 2: Number of right half-plane roots (encircled) of (10) in function of $k_{p}$ and $k_{d}$.

and $k_{d}$ (encircled). To construct this figure, we use the fact that the number of right half-plane roots can only change by roots moving over infinity at $k_{d}=1$ or crossing the imaginary axis at $\pm \jmath \omega$ for

$$
k_{p}=\frac{0.3 \sin (6 \omega)}{\omega} \text { and } k_{d}=\frac{0.3 \cos (6 \omega)}{\omega^{2}}+1 .
$$

It follows from this figure, that system (11) can only be stabilized for a derivative gain $k_{d}$ strictly larger than one. It now follows from the results in Section II that the resulting controller loses stability under arbitrarily small feedback delay, an arbitrarily close finite difference approximation and low pass filtering with an arbitrarily large cut-off frequency. Furthermore, the system can not be strongly stabilized by including a low pass filter as this filter itself destabilizes the system.

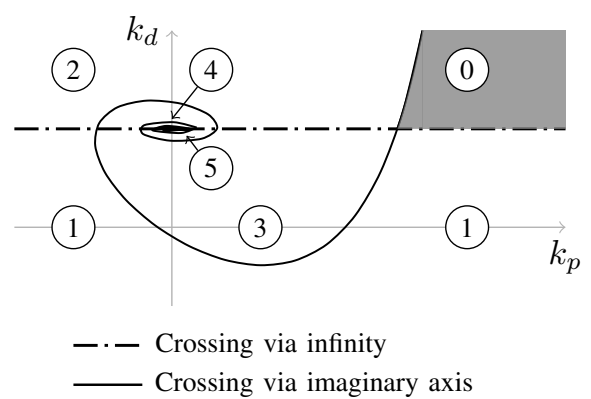

Fig. 3: Number of right half-plane roots (encircled) of (12) in function of $k_{p}$ and $k_{d}$. 


\section{Controller Design}

In this section, we describe a computational procedure to design a PD output feedback controller with low pass filtering of the derivative signal of form (7), which strongly stabilizes system (1). To synthesize such controllers, we will minimize the spectral abscissa of (3), i.e. the real part of its right-most characteristic root, in function of $k_{p}$ and $k_{d}$, under the constraint that $k_{d} c^{T} b<1$. This design procedure can be summarized as follows:

1) choose initial gains $k_{p}$ and $k_{d}$ such that $k_{d} c^{T} b<1$;

2) minimize the spectral abscissa of (3)

$$
f\left(k_{p}, k_{d}\right)=\max _{\lambda \in \mathbb{C}}\{\Re(\lambda): \Delta(\lambda)=0\}
$$

in $k_{p}$ and $k_{d}$ under the constraint $k_{d} c^{T} b<1$;

3 ) choose a sufficiently small $T$ such that the closed loop of (1) and (7) is asymptotically stable.

The constraint in step 2 ensures that the condition in Proposition 4 is fulfilled. This constraint is handled using a penalty method. More precisely, we minimize

$$
f\left(k_{p}, k_{d}\right)+\gamma \max \left(0, k_{d} c^{T} b-1\right),
$$

in $k_{p}$ and $k_{d}$. If the resulting $k_{d}$ fulfills the constraint $k_{d} c^{T} b<1$ then the design procedure is stopped. Otherwise, $\gamma$ is increased and the minimization procedure is restarted until the resulting $k_{d}$ fulfills the constraint $k_{d} c^{T} b<1$. Note, however, that optimization problem (13) is non-convex and might therefore have multiple local optima. To overcome this difficulty, we run the optimization procedure starting from several initial parameters.

Next, we illustrate the effectiveness of this design method on system (9). Starting from the unstable controller $\left(k_{p}, k_{d}\right)=(1,0)$ and $\gamma$ in (13) equal to 100 , we obtain the stabilizing feedback parameters $\left(k_{p}, k_{d}\right)=(0.2378,1.0190)$. Next, we choose $1 / T=10^{4}$ such that the closed loop of (1) and (7) is exponentially stable with a decay rate of 0.03792 . Furthermore, because $k_{d} c^{T} b=-1.0190<1$ it follows from Proposition 4 that the closed loop stability is strongly stable.

\section{CONCLUDING REMARKS}

This work focused on a certain class of implementation errors that destabilize the closed loop of a system with state delays and a PD output feedback controller, even when the size of the perturbation is infinitesimal. Under certain conditions on the derivative feedback gain $k_{d}$, it was then shown that by adding a low filter pass with a sufficiently large cut-off frequency, the closed loop system can preserve its stability under sufficiently small perturbations of this class.

However, when assessing the real-life stability of the closed loop system, also other kinds of perturbations, such as modelling errors, which typically can not be made arbitrarily small, have to be taken into account. In contrast to the implementation errors on which we focused here, these perturbations typically no longer destabilize the closed loop when made arbitrarily small. Nevertheless, it is still possible for very small (but not infinitesimal) perturbations of this kind to significantly change the location of the characteristic roots; think of the roots of Wilkinson's polynomial [18]. Hence, the spectral abscissa and as a consequence the behavior of the real life system may significantly differ from the prediction by the model if such perturbations are present. To address these kind of perturbations, the pseudo-spectral framework can be used. For example, instead of minimizing the spectral abscissa of the nominal model, one could amend the model with a family of perturbations and consider more robust performance measures such as the pseudo-spectral abscissa or the distance to instability.

\section{REFERENCES}

[1] W. Michiels, T. Vyhlídal, H. Huijberts, and H. Nijmeijer, "Stabilizability and Stability Robustness of State Derivative Feedback Controllers," SIAM Journal on Control and Optimization, vol. 47, no. 6, pp. 3100$3117,2009$.

[2] T. T. Georgiou and M. C. Smith, "w-Stability of feedback systems," Systems \& Control Letters, vol. 13, no. 4, pp. 271 - 277, 1989.

[3] P. Appeltans, S.-I. Niculescu, and W. Michiels, "Analysis and Design of Strongly Stabilizing PID Controllers for Time-Delay Systems," arXiv:2009.02924 [math.OC], 2020.

[4] J. Willems, The Analysis of Feedback Systems. Cambridge, MA: MIT Press, 1971.

[5] J. Barman, F. Callier, and C. Desoer, " $L^{2}$-stability and $L^{2}$-instability of linear time-invariant distributed feedback systems perturbed by a small delay in the loop," IEEE Transactions on Automatic Control, vol. 18 , no. 5 , pp. 479-484, 1973.

[6] H. Logemann, R. Rebarber, and G. Weiss, "Conditions for robustness and nonrobustness of the stability of feedback systems with respect to small delays in the feedback loop," SIAM Journal on Control and Optimization, vol. 34, no. 2, pp. 572-600, 1996.

[7] H. Logemann, "Destabilizing effects of small time delays on feedbackcontrolled descriptor systems," Linear Algebra and its Applications, vol. 272, no. 1, pp. $131-153,1998$.

[8] J. K. Hale and S. M. Verduyn Lunel, "Strong stabilization of neutral functional differential equations," IMA Journal of Mathematical Control and Information, vol. 19, no. 1 and 2, pp. 5-23, 2002.

[9] H. Khalil, "On the robustness of output feedback control methods to modeling errors," IEEE Transactions on Automatic Control, vol. 26 , no. 2, pp. 524-526, 1981.

[10] M. Vidyasagar, "Robust stabilization of singularly perturbed systems," Systems \& control letters, vol. 5, no. 6, pp. 413-418, 1985.

[11] R. Sipahi, G. Arslan, and S.-I. Niculescu, "Some remarks on control strategies for continuous gradient play dynamics," in Proceedings of the 45th IEEE Conference on Decision and Control, 2006, pp. 19661971.

[12] D. Lee and M. W. Spong, "Passive bilateral teleoperation with constant time delay," IEEE Transactions on Robotics, vol. 22, no. 2, pp. 269281, 2006.

[13] B. Liacu, A. Taha Koru, H. Özbay, S.-I. Niculescu, and C. Andriot, "Optimizing low-order controllers for haptic systems under delayed feedback," Control Engineering Practice, vol. 21, no. 5, pp. $655-$ 668, 2013.

[14] M. Vidyasagar, Control System Synthesis: A Factorization Approach (Part I). Morgan \& Claypool Publishers, 2011.

[15] W. Michiels and S.-I. Niculescu, Stability, Control, and Computation for Time-Delay Systems. Philadelphia, PA: Society for Industrial and Applied Mathematics, 2014.

[16] N. D. Hayes, "Roots of the transcendental equation associated with a certain difference-differential equation," Journal of the London Mathematical Society, vol. s1-25, no. 3, pp. 226-232, 1950.

[17] E. C. Titchmarsh, The theory of functions (2nd edition), pp. 116-118, Oxford: Oxford University Press, 1939.

[18] J. H. Wilkinson, "The evaluation of the zeros of ill-conditioned polynomials. Part I," Numerische Mathematik, vol. 1, no. 1, pp. 150$166,1959$. 